\title{
AUMENTO DE PRODUTIVIDADE DA FROTA DE TRANSPORTE DA MINA OESTE*
}

\section{Resumo}

\author{
Frederico Augusto Rosa do Carmo ${ }^{1}$ \\ Adílson Cesar Melo de Paula ${ }^{2}$ \\ Alessandro Domingos Vilela da $\mathrm{Cruz}^{3}$ \\ Alexandre Vieira Magalhães Santos ${ }^{4}$ \\ Bruno Moreira ${ }^{5}$ \\ Frederico Ibrahim Horta ${ }^{6}$ \\ Jefferson de Almeida Quadros ${ }^{7}$ \\ Leandro Campos de Lima $^{8}$
}

As estradas de mineração influenciam diretamente nos custos de operação de mina e manutenção de equipamentos. A equipe de planejamento de lavra em conjunto com a equipe de operação de mina buscando a melhoria de seus indicadores de custos e produtividade realizaram várias melhorias no perfil de transporte da Mina Oeste. Para realização deste trabalho foram mapeados e projetados novos trechos da mina que poderiam ser melhorados em termos de inclinação de rampa, subidas, descidas e curvas. A realização destas melhorias, em conjunto com correções de drenagem e forragem das pistas, possibilitou a alteração do limite de velocidade dos caminhões fora-de-estrada em alguns trajetos de 40 para $50 \mathrm{~km} / \mathrm{h}$. Como resultado destas alterações, obteve-se um ganho de $18 \%$ na produtividade da frota de transporte e uma redução de $13 \%$ nos custos de operação.

Palavras-chave: Produtividade; Planejamento de Lavra; Operação de Mina; Redução de Custos.

\section{INCREASE IN PRODUCTIVITY OF OESTE MINE TRANSPORTATION FLEET Abstract}

Mining roads directly influence the costs of mine operation and equipment maintenance. Mining planning team together with mine operation team seeking to improve their cost and productivity indicators, have made several improvements in roads segments to the Oeste mine. For the achievement of this work were mapped and projected new segments in the mine that could be improved in terms of incline ramp, rises, declines and curves. The realization of these improvements together with corrections of drainage and forage of the roads, allowed the change of the speed limit of the trucks off-road in some routes of 40 to $50 \mathrm{~km} / \mathrm{h}$. As result of these changes, there was an $18 \%$ increase in the productivity of the transport fleet and a $13 \%$ reduction in operating costs.

Keywords: Productivity; Mining planning; Mine operation; Cost reduction.

\footnotetext{
Gerente de Planejamento de Lavra e Geotecnia, Mineração Usiminas, Minas Gerais, Brasil. Gerente de Operação de Mina, Mineração Usiminas, Minas Gerais, Brasil.

Técnico de Mineração II, Mineração Usiminas, Minas Gerais, Brasil.

4 Engenheiro Especialista, Gerência de Planejamento de Lavra e Geotecnia, Mineração Usiminas, Minas Gerais, Brasil.

5 Engenheiro de Processos, Gerência de Operação, Mineração Usiminas, Minas Gerais, Brasil.

6 Engenheiro Sênior, Gerência de Planejamento de Lavra e Geotecnia, Mineração Usiminas, Minas Gerais, Brasil.

7 Técnico de Mineração II, Gerência de Planejamento de Lavra e Geotecnia, Mineração Usiminas, Minas Gerais, Brasil.

8 Técnico de Mineração II, Gerência de Planejamento de Lavra e Geotecnia, Mineração Usiminas, Minas Gerais, Brasil.
} 


\section{INTRODUÇÃO}

Em uma lavra a céu aberto, a maior parte dos custos da operação de mina é decorrente das etapas de carregamento e transporte, que pode envolver tanto a extração do minério, quanto a remoção do estéril, material sem valor econômico cuja remoção se dá devido à necessidade de liberação de minério. Nestas etapas os materiais são alocados predominantemente em caminhões, sendo o minério destinado à etapa de beneficiamento e o estéril disposto em pilhas.

A produtividade das frotas de transporte depende de diversos fatores tais como tempo de fila dos caminhões nas frentes de lavra e nos pontos de basculamento, distância média entre os pontos iniciais e finais dos ciclos de transporte e velocidade média nas vias. Para que se tenha uma boa política de maximização desta produtividade é essencial que os acessos estejam perfeitamente adequados aos equipamentos selecionados para a operação.

Com base nestes princípios, o objetivo geral do trabalho foi buscar a melhoria das vias utilizadas para o transporte dos materiais de forma a proporcionar uma redução dos custos de operação com o aumento da produtividade da frota.

\section{MATERIAIS E MÉTODOS}

O perfil escolhido para o projeto dos acessos e rampas de uma mina está intimamente ligado ao desenvolvimento da lavra e das condições geológicas e geotécnicas das áreas atravessadas. O perfil também deve garantir uma uniformidade das operações de transporte.

Para o caso específico das rampas a declividade é expressa em porcentagem, que corresponde à relação entre a altura do banco e a extensão horizontal das rampas. Quanto maior a porcentagem da declividade da rampa, maior será a perda de velocidade do equipamento ao longo do percurso. O tempo de percurso dos caminhões nas rampas dependerá também da relação peso / potência. Assim, equipamentos com a mesma relação peso / potência terão 0 mesmo comportamento. Com base nisso, estipulou-se uma inclinação ideal de $6 \%$ para minimização das perdas de velocidade, sendo $10 \%$ a máxima permitida para os projetos das rampas.

Após estas definições, mapeou-se ao longo da mina regiões onde era possível suavizar o perfil de transporte, buscando minimizar o impacto da declividade de subidas e descidas na velocidade média de percurso dos equipamentos e eliminando pontos onde a declividade ultrapassava os $10 \%$ permitidos. Foram realizados cortes com remoção de estéril em alguns trechos - sempre de acordo com as etapas de desenvolvimento da mina - e aterro em outros locais através da formação de estoque de minérios de baixo teor para futura destinação à etapa de beneficiamento. Para a escolha das áreas atravessadas pelo traçado principal da mina, levou-se em consideração o desenvolvimento da lavra ao longo do ano. Esta etapa ocorreu no período de agosto de 2015 a fevereiro de 2016. 




Figura 1. Perfil de transporte trecho 1 antes e depois com diminuição da distância e inclinação.

\begin{tabular}{|l|l|}
\hline \\
\\
Perfil de transporte em 29/07/2015
\end{tabular}

Figura 2. Perfil de transporte trecho 2 antes e depois com diminuição da distância e inclinação.

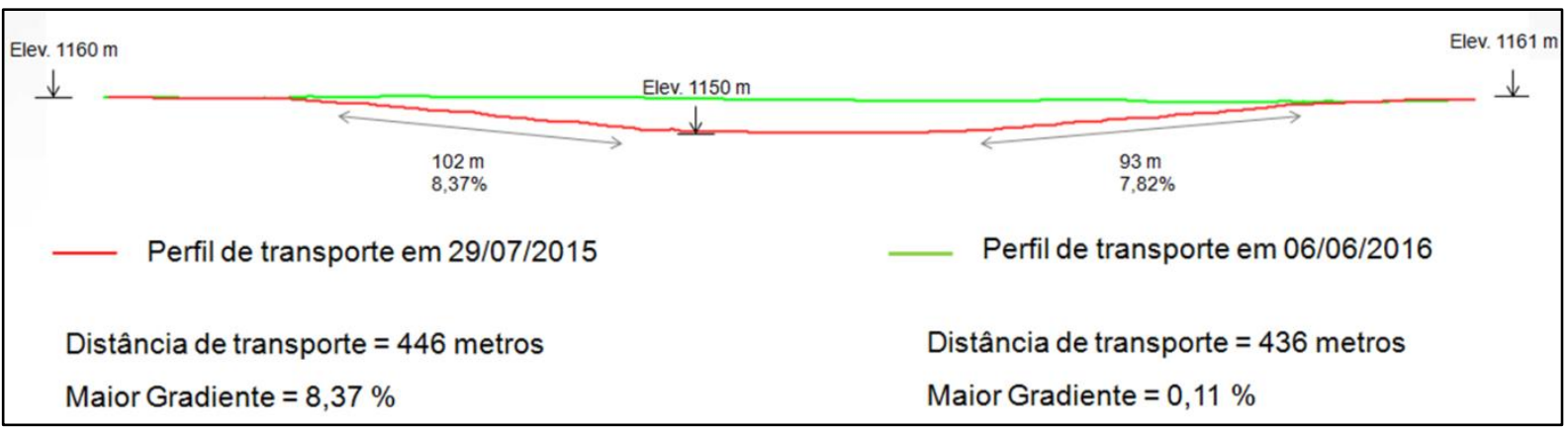

Figura 3. Perfil de transporte trecho 3 antes e depois com diminuição da distância e inclinação.

A etapa seguinte do projeto, ocorrida de fevereiro a março de 2016, correspondeu a melhorias de infraestrutura realizadas ao longo do trajeto como: forragem das estradas com material extraído da mina que garantisse uma boa interação pneu-solo e drenagem com inclinação de 2 a $4 \%$ para escoamento da água pelas pistas no sentido transversal. 

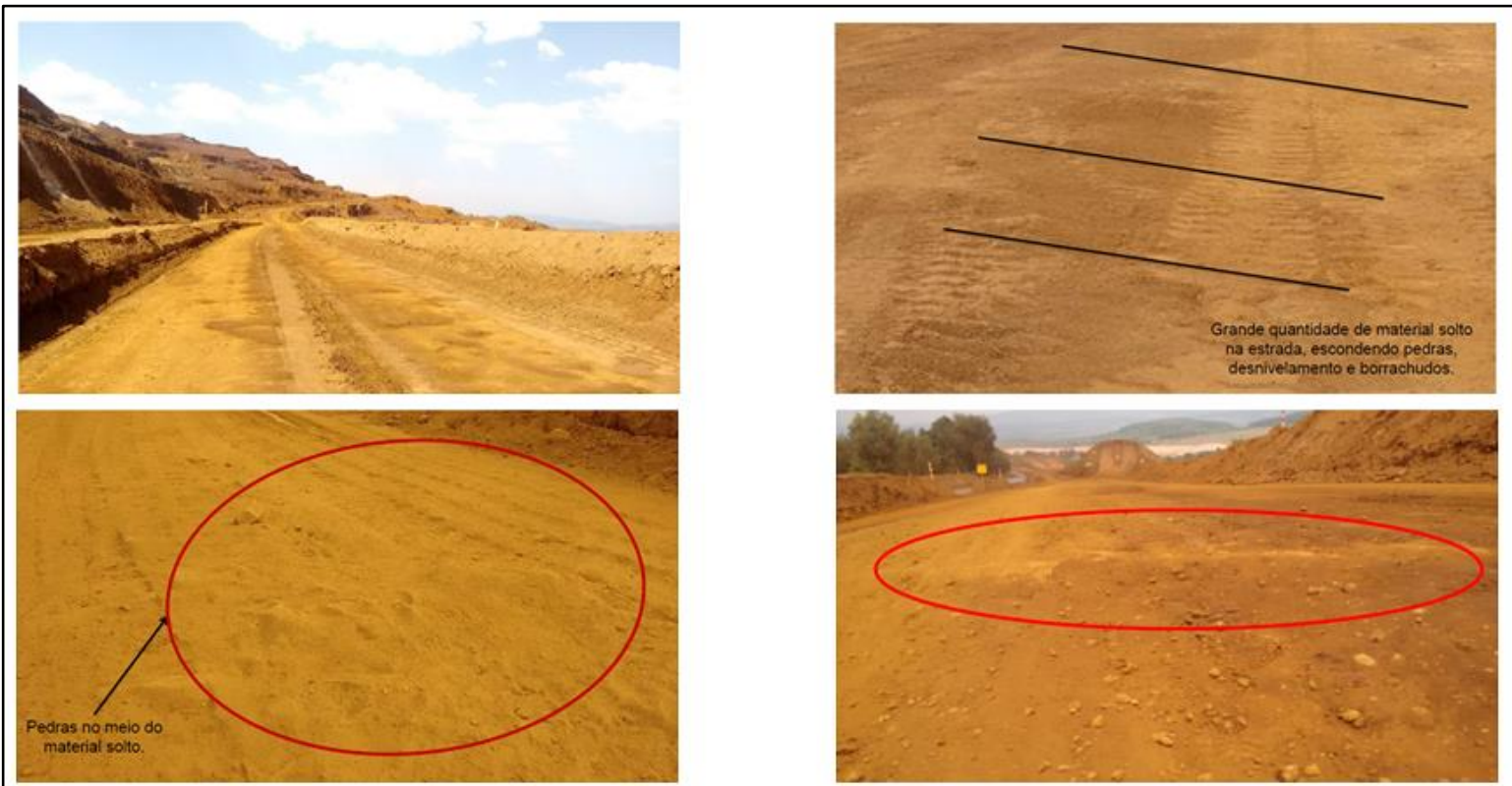

Figura 4. Estradas da mina antes das melhorias de infraestrutura.

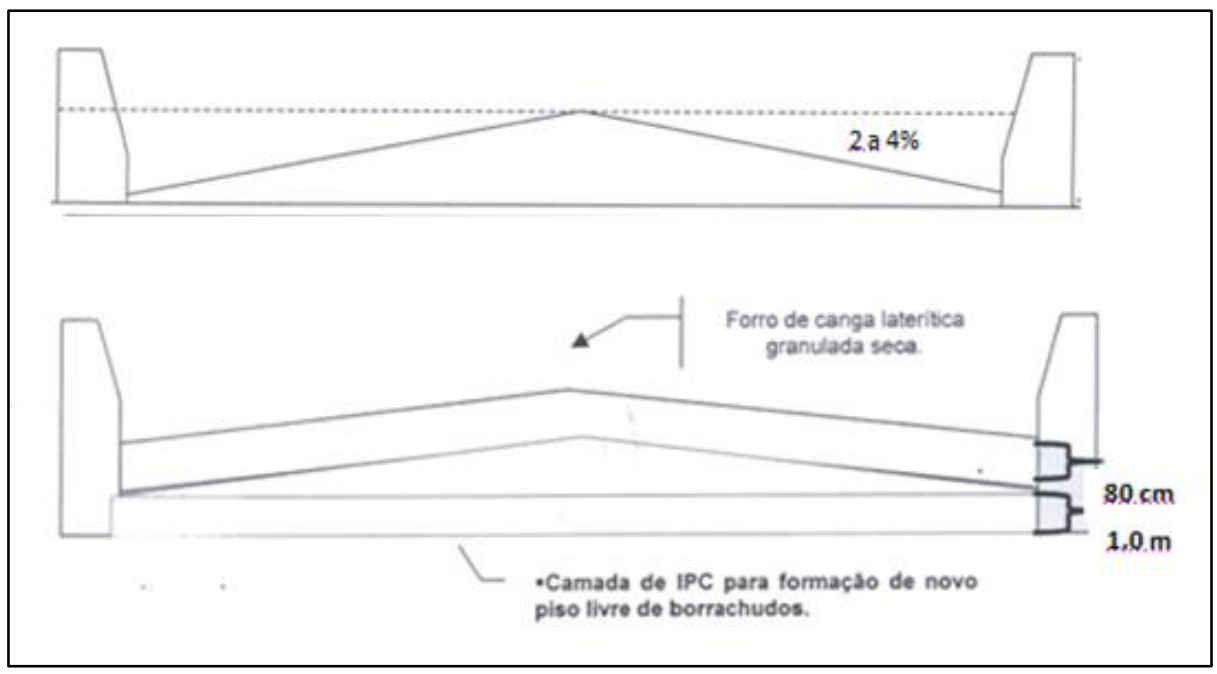

Figura 5. Projeto de melhoria do perfil das estradas da mina.

Após a conclusão de todas estas melhorias foi possível criar uma via extensa, sem curvas acentuadas e sem subidas e descidas onde alterou-se a velocidade máxima de 40 para $50 \mathrm{~km} / \mathrm{h}$. 


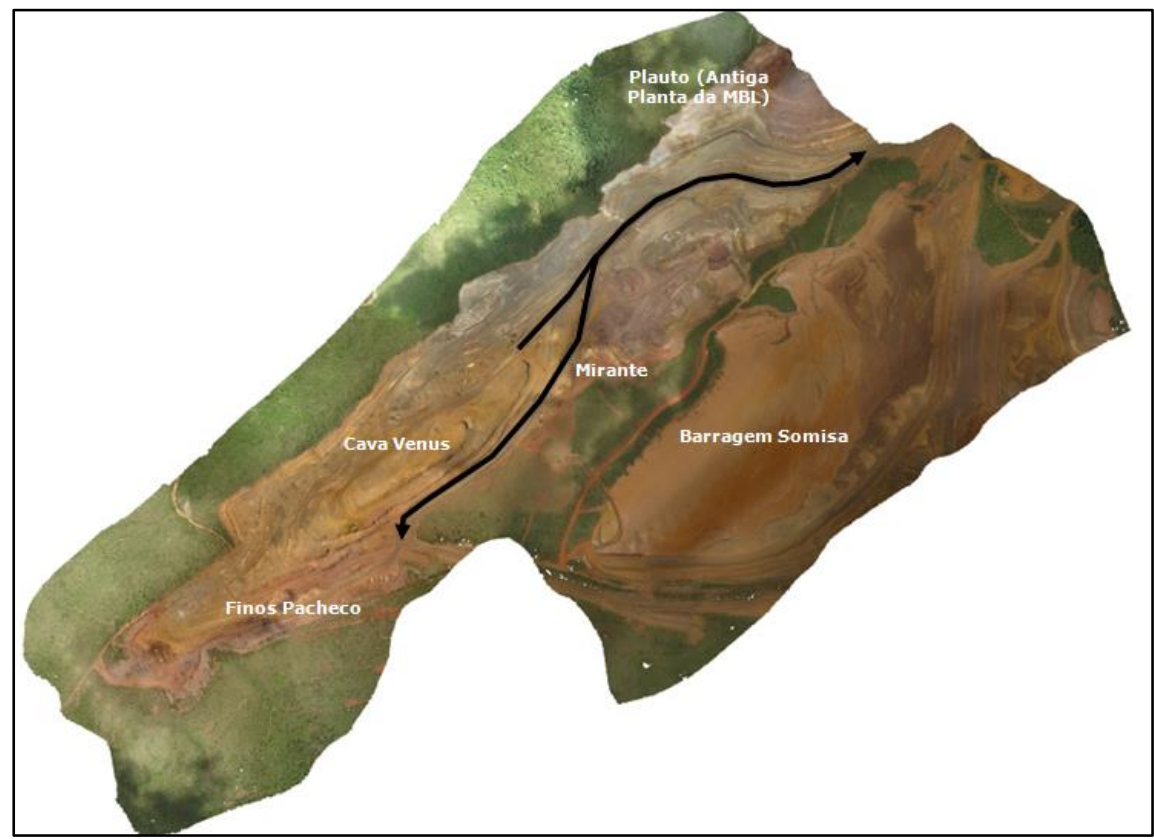

Figura 6. Trecho da mina onde ocorreram as melhorias no perfil de transporte.

\section{RESULTADOS E DISCUSSÃO}

Os critérios adotados para a análise dos resultados foram: produtividade, a velocidade média dos caminhões, a distância média de transporte (DMT), e o consumo específico $(\mathrm{l} / \mathrm{h})$.

Foram avaliados dados históricos desde o ano de 2013 até o período de fevereiro de 2017 da frota de caminhões fora de estrada com capacidade de 95 toneladas. $O$ tipo de equipamento e suas especificações foram as mesmas durante todo este período.

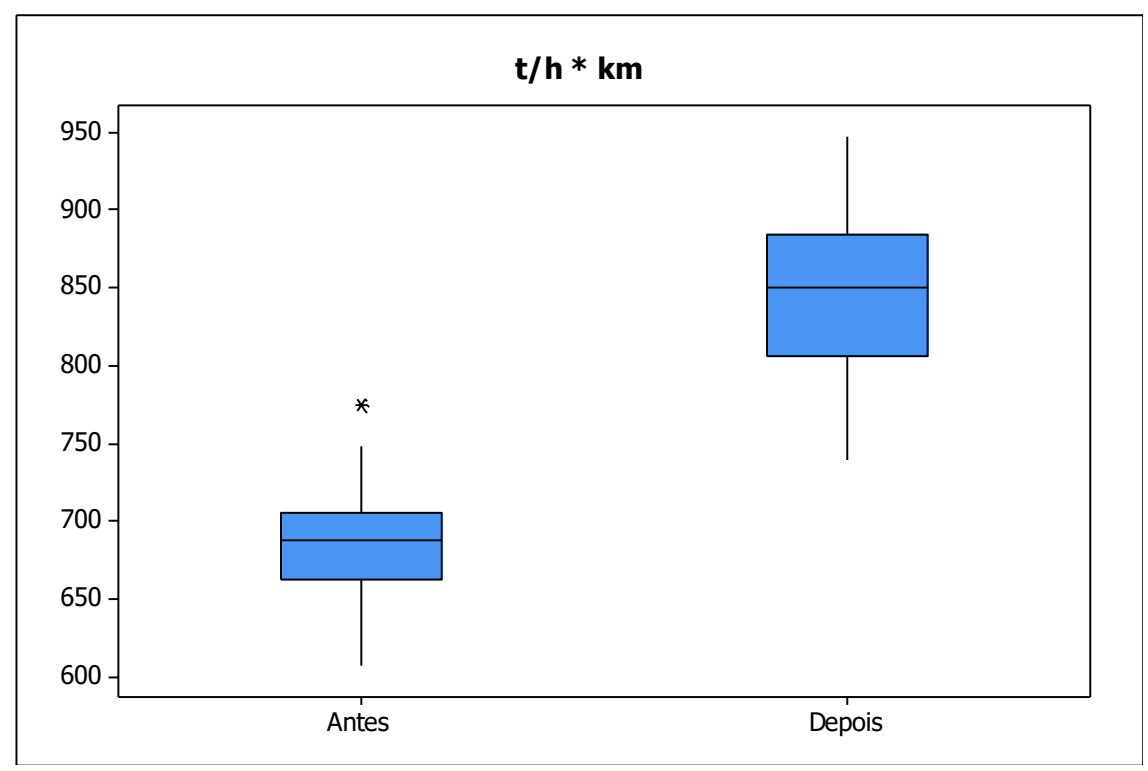

Figura 7. Parâmetros de $t / h^{\star} k m$ antes e depois da mudança na velocidade máxima de transporte, considerando o período de Abril de 2013 a Fevereiro de 2017. 
Em situações de operações ideais, com carga média de transporte constante, a produtividade será inversamente proporcional à distância média de transporte, ou seja, quanto menor a distância maior será a produtividade da frota. Dessa forma, para anular o efeito da distância sobre a produtividade, optou-se por multiplicar os dois índices. Temos assim os parâmetros de $\mathrm{t} / \mathrm{h}$ * $\mathrm{km}$, conforme boxplot representado na Figura 5. Comparando-se o período antes e depois da mudança na velocidade máxima de transporte, observa-se uma melhoria significativa do desempenho da frota.

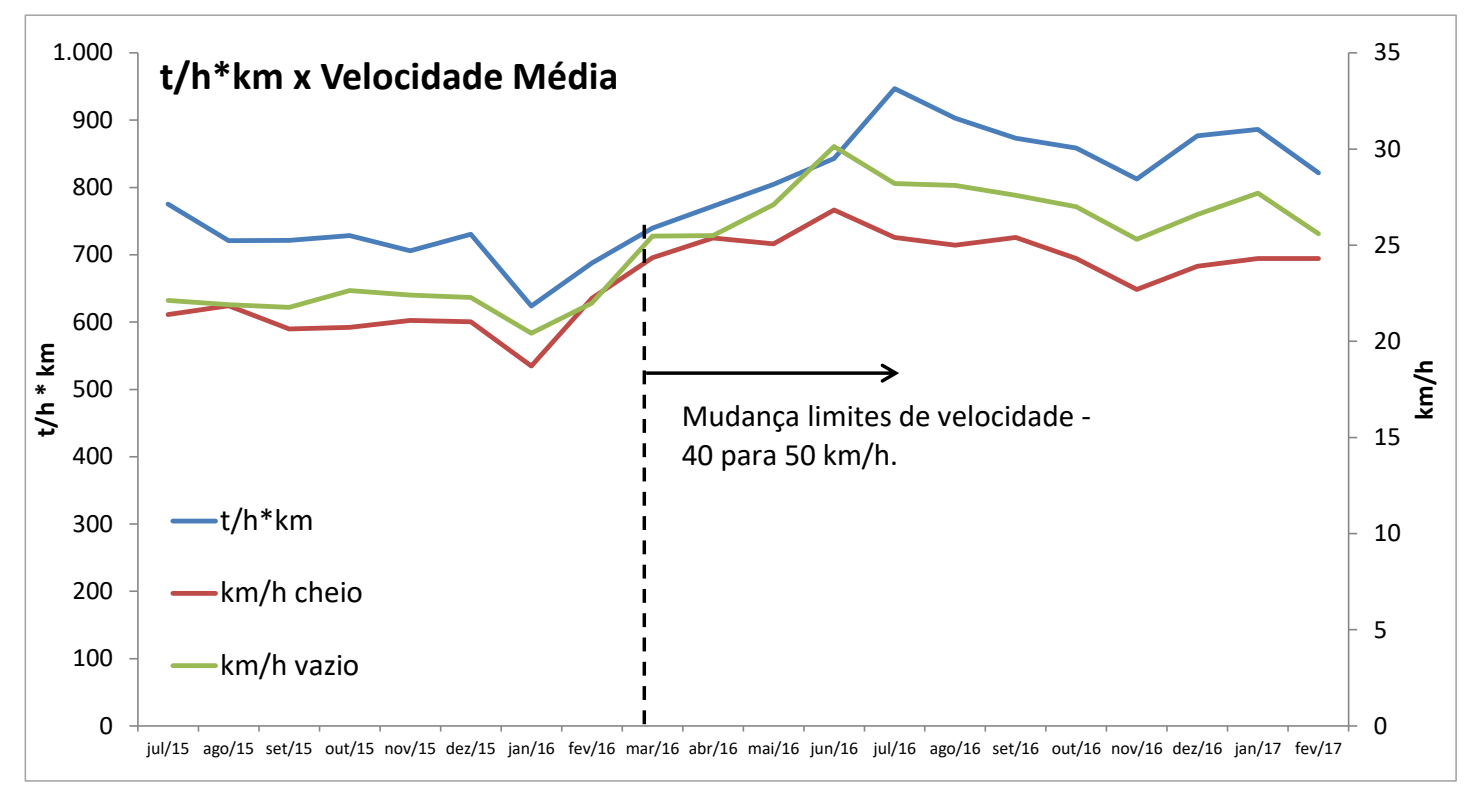

Figura 8. Relação entre o índice t/h $\mathrm{km}$ e as velocidades médias da frota de transporte.

No gráfico acima é possível observar de forma direta a relação entre a velocidade média dos caminhões com o índice de $t / h^{*} \mathrm{~km}$ no período de julho de 2015 a fevereiro de 2017, mostrando a influência da alteração da velocidade máxima no aumento de produtividade da frota. Outra análise realizada foi através do Coeficiente de Pearson - mede o grau de correlação linear entre duas variáveis quantitativas onde se verificou a correlação entre os dados de velocidade e $t / h^{*} \mathrm{~km}$. Este coeficiente, normalmente representado pela letra $r$, assume valores entre -1 e 1 , sendo quanto maior o valor de $r$ (negativo ou positivo), mais forte a associação. $O$ valor encontrado para este caso foi de $r=0,787$; mostrando uma forte correlação entre os valores analisados.

Para aferir os ganhos com o projeto simulou-se a produtividade utilizando os dados históricos como tendência. Chegamos assim ao gráfico abaixo. 




Figura 9. Índice t/hkm real versus simulado de abril de 2013 a fevereiro de 2017.

O gráfico mostra perfeitamente o comparativo entre o desempenho da frota com a mudança de velocidade e o caso simulado com a velocidade anterior praticada.

Tabela 1. Dados de produtividade e ciclo de transporte entre fevereiro de 2016 a janeiro de 2017

\begin{tabular}{|c|c|c|c|c|c|c|c|c|c|c|c|c|}
\hline $\begin{array}{l}\text { MINA OESTE } \\
\text { (RDP490) }\end{array}$ & $\begin{array}{l}\text { PROD. } \\
(\mathrm{t} / \mathrm{h})\end{array}$ & $\begin{array}{c}\text { EFICIENCIA } \\
\text { OPERACIONAL } \\
(\%)\end{array}$ & DMT (km) & $\begin{array}{c}\text { CARGA } \\
\text { MÉDIA (t) }\end{array}$ & $\begin{array}{l}\text { MANOBRA } \\
\text { NA } \\
\text { DESCARGA } \\
\text { (s) }\end{array}$ & $\begin{array}{c}\text { MANOBRA } \\
\text { NA CARGA } \\
(s)\end{array}$ & $\begin{array}{l}\text { TEMPO } \\
\text { DE CARGA } \\
\text { (s) }\end{array}$ & $\begin{array}{c}\text { FILA } \\
\text { NA CARGA } \\
\text { (s) }\end{array}$ & $\begin{array}{l}\text { VELOCIDADE } \\
\text { CARREGADO } \\
(\mathrm{km} / \mathrm{h})\end{array}$ & $\begin{array}{c}\text { VELOCIDADE } \\
\text { VAZIO } \\
(\mathrm{km} / \mathrm{h})\end{array}$ & $\begin{array}{c}\text { FILA } \\
\text { DESCARGA } \\
\text { (s) }\end{array}$ & $\begin{array}{c}\text { TEMPO } \\
\text { DESCARGA } \\
\text { (s) }\end{array}$ \\
\hline V.Máx (40km/h ) & 334,43 & $95,71 \%$ & 2,08 & 95,25 & 24,35 & 19,97 & 149,55 & 27,16 & 19,21 & 21,72 & 0,75 & 34,81 \\
\hline V.Máx (50 km/h ) & 395,91 & $95,71 \%$ & 2,08 & 95,25 & 24,35 & 19,97 & 149,55 & 27,16 & 24,09 & 26,23 & 0,75 & 34,81 \\
\hline
\end{tabular}

Na tabela acima se observa um ganho de $18 \%$ nos dados de produtividade $(\mathrm{t} / \mathrm{h})$ ao se comparar as situações com velocidade máxima de 40 e 50 km/h.

O ponto negativo associado à mudança de velocidade foi o aumento do consumo específico (litros por hora), conforme gráfico abaixo. Por outro lado, o aumento do consumo específico não se refletiu em aumento nos custos, já que a frota passou a fazer o mesmo percurso em menor tempo.

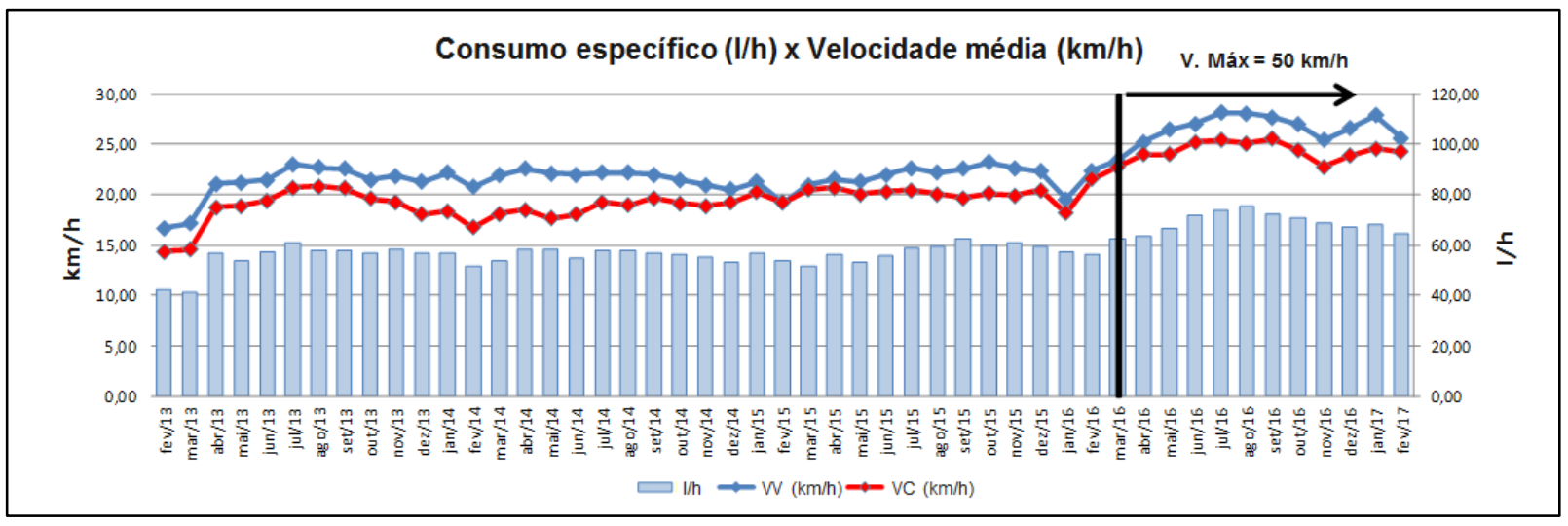

Figura 10. Consumo específico (I/h) e velocidade média da frota de fevereiro de 2013 a fevereiro de 2017. 
Comparando-se a produtividade alcançada com as melhorias, com a produtividade anteriormente praticada, verificou-se uma redução 13\% nos custos de operação. Foram observados também ganhos intangíveis como: melhoria nas condições de trabalho para os operadores, menor tempo de parada por pista escorregadia e maior segurança no tráfico de mina.

\section{CONCLUSÃO}

Em cenários cada vez mais competitivos na mineração, é de grande importância a realização de projetos que envolvam a redução de custos e a maximização dos ativos. As melhorias em índices operacionais atrelados ao bom desenvolvimento da lavra se tornam cruciais para a manutenção do negócio.

A produtividade da frota apresentou melhores resultados após os trabalhos de melhoria nos perfis de transporte e o aumento do limite de velocidade de 40 para 50 $\mathrm{km} / \mathrm{h}$. Tivemos um aumento de $18 \%$ na produtividade da frota e uma economia de $18 \%$ nos custos de operação durante o período de fevereiro de 2016 a janeiro de 2017. Foi possível demonstrar que essa melhoria estava associada diretamente com o aumento de velocidade e não a outros parâmetros como a DMT.

O trabalho mostrou que é possível associar o bom desenvolvimento da lavra com melhoria nos índices operacionais através de uma forte sinergia entre as áreas de planejamento de lavra e operação de mina.

\section{REFERÊNCIAS}

1 Pinto EB. Despacho de caminhões em mineração usando lógica nebulosa, visando ao atendimento simultâneo de políticas excludentes. Escola de Engenharia da Universidade Federal de Minas Gerais. Belo Horizonte, MG. Brasil. 2007.

2 Macedo EL. Noções de topografia para projetos topográficos. 2008. [acesso em 03 jan. 2012];14:1-5. Disponível em: http://www.topografiageral.com/Curso/capitulo\%2014.php

3 Andrade, E.L. Introdução à pesquisa operacional. Livros Técnicos e Científicos Editora. S.A. 2 ${ }^{\text {a }}$ Edição. Rio de Janeiro. 1990.

4 Lage ER. Utilização do método de corte e enchimento no fechamento de uma mina a céu aberto: proposta de metodologia para o caso da Mina de Alegria 3, 4 e 5 da Samarco Mineração S.A. Universidade Federal de Ouro Preto: Curso de PósGraduação em Engenharia Mineral. Ouro Preto, MG. Brasil. 2001.

5 Santos SS. Metodologia de planejamento de lavra de curto prazo - uma abordagem de otimização e gestão. Universidade Federal de Ouro Preto: Curso de Pós-Graduação em Sistemas Minero Metalúrgicos. Ouro Preto, MG. Brasil. 2014. 